\title{
Absence of placental growth factor blocks dextran sodium sulfate-induced colonic mucosal angiogenesis, increases mucosal hypoxia and aggravates acute colonic injury
}

\author{
Pieter Hindryckx ${ }^{1,5}$, Anouk Waeytens ${ }^{1,5}$, Debby Laukens ${ }^{1}$, Harald Peeters ${ }^{1}$, Jacques Van Huysse ${ }^{2}$, \\ Liesbeth Ferdinande ${ }^{2}$, Peter Carmeliet ${ }^{3,4}$ and Martine De Vos ${ }^{1}$
}

\begin{abstract}
Angiogenesis has recently been described as a component of inflammatory bowel disease. Placental growth factor (PIGF), a vascular endothelial growth factor (VEGF) homologue, establishes its angiogenic capacity under pathophysiological conditions. We investigated the function of PIGF in experimental models of acute colitis. Acute colonic damage was induced in PIGF knock-out $\left(^{-1-}\right)$ mice and PIGF wild-type $\left(^{+/+}\right)$mice by dextran sodium sulfate (DSS) and trinitrobenzenesulfonic acid (TNBS). The concentrations of PIGF and VEGF were measured in distal colonic lysates using an enzyme-linked immunosorbent assay. Colonic injury was evaluated by assessing colon length, colonocyte apoptosis (by terminal dUTP nick-end labeling), colonic cytokine production and histological score. Infiltration of polymorphonuclear cells was determined by assaying myeloperoxidase (MPO) activity. In a separate experiment, recombinant PIGF was administered to PIGF ${ }^{-1-}$ mice by adenoviral transfer before DSS administration. Mucosal vascularization was quantified by computerized morphometric analysis of CD31-stained distal colonic sections. Colonic mucosal hypoxia was visualized by pimonidazole staining. Both VEGF and PIGF were upregulated during acute colitis. In addition, compared with PIGF ${ }^{+1+}$ controls, $\mathrm{PIGF}^{-1-}$ mice showed a significant increase in weight loss and colonic shortening during both DSS and TNBS colitis. This correlated with enhanced colonocyte apoptosis, elevated colonic cytokine levels and increased histological damage score, but not with enhanced inflammatory cell infiltration (MPO activity). The increased morbidity of PIGF ${ }^{-1-}$ mice during DSS colitis was preventable by adenovirus (Ad)-mediated overexpression of PIGF. After the administration of DSS, strongly reduced mucosal angiogenesis was observed in $\mathrm{PIGF}^{-1-}$ mice compared with PIGF ${ }^{+/+}$ mice. This was associated with an early increase in intestinal epithelial pimonidazole accumulation in $\mathrm{PIGF}^{-1-}$ mice, suggesting a function of enhanced epithelial hypoxia in the observed differences between the two groups. In summary, our data show that the absence of PIGF strongly inhibits mucosal intestinal angiogenesis in acute colitis, which is associated with an early increase in intestinal epithelial hypoxia and aggravation of the course of the disease. Laboratory Investigation (2010) 90, 566-576; doi:10.1038/labinvest.2010.37; published online 8 February 2010
\end{abstract}

KEYWORDS: angiogenesis; experimental colitis; inflammatory bowel disease; intestinal mucosal hypoxia; placental growth factor; vascular endothelial growth factor

Inflammatory bowel diseases (IBD, the major types being Ulcerative Colitis and Crohn's Disease) are chronic, relapsing inflammatory conditions of the gut causing intestinal mucosal damage. Their etiology is incompletely understood. Angiogenesis, defined as the growth of new blood vessels from pre-existing ones, has recently been described as a component of IBD. An increase in vascularization and an active angiogenic profile is seen in the colonic mucosa of patients with active IBD compared with healthy controls or patients with inactive disease. ${ }^{1}$ In addition, intestinal angiogenesis seems to be directly correlated with inflammation in experimental murine models of colitis. ${ }^{2,3}$

\footnotetext{
${ }^{1}$ Department of Gastroenterology, Ghent University, Gent, Belgium; ${ }^{2}$ Department of Pathology, Ghent University, Gent, Belgium; ${ }^{3}$ Vesalius Research Center, VIB, Leuven, Belgium and ${ }^{4}$ Vesalius Research Center, KU Leuven, Leuven, Belgium

Correspondence: Dr P Hindryckx, MD, Department of Gastroenterology, Ghent University Hospital, De Pintelaan 185 3K12IE, Gent B-9000, Belgium. E-mail: pieter.hindryckx@ugent.be

${ }^{5}$ These two authors contributed equally to this work.

Received 17 August 2009; revised 16 December 2009; accepted 3 January 2010
} 
Placental growth factor (PlGF), a member of the vascular endothelial growth factor (VEGF) family, has received little attention in angiogenesis research. However, recent genetic studies in mice have revealed an angiogenic function for PlGF that is restricted to pathophysiological conditions. ${ }^{4}$ In contrast to VEGF-A, the main isoform of the VEGF family (hereafter referred to as 'VEGF'), PlGF knockout is not embryonic lethal and leaves physiological angiogenesis unaffected. As a result, antibodies directed against PlGF are attractive candidates for the treatment of conditions associated with pathological angiogenesis, such as neoplastic disease and arthritis. ${ }^{5,6}$ However, 'pathophysiological' does not always mean 'detrimental'. PIGF has been shown to have beneficial effects in wound healing and ischemic cardiac disease. ${ }^{4,7}$

The function of angiogenesis in IBD pathogenesis remains poorly understood because of its opposing effects. On one hand, angiogenesis is a crucial process to supply oxygen and nutrients to promote healing of the damaged mucosa. On the other hand, angiogenesis may sustain inflammation by enhancing the influx of inflammatory cells.

The function of PIGF in experimental colitis remains to be established. Therefore, the aim of this study was to investigate whether PlGF is involved in two murine models of IBD: dextran sodium sulfate (DSS)-induced colitis and trinitrobenzenesulfonic acid (TNBS)-induced colitis.

\section{MATERIALS AND METHODS}

Mice

In total, $49129 / \mathrm{Sv}$ PlGF wild-type $\left({ }^{+/+}\right)$mice and 55 ageand weight-matched PIGF knock-out $\left(^{-l-}\right)$ mice were kindly provided by the Flanders Interuniversity Institute for Biotechnology (VIB, Leuven, Belgium). All mice possessed a written health certificate. They were kept under standard conditions and treated according to institutional animal health care and use guidelines. The study was approved by the institutional review board.

\section{Induction of Acute Colonic Injury and Treatment} In a first experiment, $34 \mathrm{PlGF}^{+/+}$and $32 \mathrm{PlGF}^{-1-}$ mice were fed ad libitum with DSS (MP Biomedicals, California, USA) dissolved in the drinking water for 7 days to induce acute colonic injury. ${ }^{9}$ The optimal DSS concentration was screened first and set at $4 \%$. DSS solutions were monitored to ensure equal consumption between $\mathrm{PlGF}^{+/+}$and $\mathrm{PlGF}^{-1-}$ mice.

In a separate experiment, $\mathrm{PlGF}^{-/-}$mice were given an IV injection with $1 \times 10^{9}$ plaque-forming units $(\mathrm{pfu})$ of an adenovirus expressing PlGF (AdPlGF, $N=6$ ) or a control adenovirus (AdRR5, $N=6$ ) into the tail vein, 1 day before administration of DSS. ${ }^{10}$ The disease course was compared with $\mathrm{PlGF}^{+/+}$mice, which received AdRR5 $(N=6)$.

Finally, TNBS colitis was induced in seven $\mathrm{PlGF}^{-1-}$ and six $\mathrm{PlGF}^{+/+}$mice by intrarectal application of $100 \mu \mathrm{l}$ of a $1: 1$ solution of absolute ethanol and 5\% TNBS, as described earlier. ${ }^{11}$ Control mice (four $\mathrm{PlGF}^{-1-}$ and three $\mathrm{PlGF}^{+/+}$ mice) received the same volume of $50 \%$ ethanol alone.

\section{Clinical Observation and Sampling}

During the intial DSS experiment, body weight, digestive bleeding and stool consistency were determined daily. For detection of fecal occult blood, Coloscreen ${ }^{\mathrm{R}}$ lab packs were used (Helena Laboratories, Texas, USA). Clinical scores were determined as described earlier. ${ }^{12}$

Sampling was performed at day 0 (baseline), day 3 (early onset of injury), day 7 (full-blown acute injury) and day 11 (early recovery). ${ }^{9}$

During the DSS experiment that followed adenoviral transfection, body weight was monitored daily and tissue sampling was performed at day 9 .

During the TNBS experiment, body weight was monitored daily and tissue sampling was performed at day 3 .

All mice were anesthetized (20\% Xylazine, 80\% Ketamine) before killing. Colon length was measured as an indirect morphometric marker of colonic injury. Tissue prelevation was performed in a standardized manner.

\section{Histological Inflammation}

Sections of the distal colon were cut into sections that were $5 \mu \mathrm{m}$ thick and stained with hematoxylin and eosin (H\&E). Colonic epithelial injury (ulcerations and destruction of crypt architecture) and inflammation (mucosal and submucosal inflammatory cell infiltration) were blindly scored by two independent observers (one of whom was a pathologist). Scores were determined as reported earlier. ${ }^{12}$

\section{Myeloperoxidase Assay}

The myeloperoxidase (MPO) assay was performed as described earlier. ${ }^{13}$ In short, distal colon samples were suspended in $50 \mathrm{mM}$ potassium phosphate buffer ( $\mathrm{pH}$ 6.0) containing $0.5 \%$ hexadecyltrimethylammonium bromide (Sigma, Missouri, USA) followed by sonication on ice for $15 \mathrm{~s}$. Suspensions were then freeze-thawed three times and the supernatant was separated from the solid phase by centrifugation at $16000 \mathrm{~g}$ for $20 \mathrm{~min}$. A total of $10 \mu \mathrm{l}$ of the supernatant was mixed with $140 \mu \mathrm{l}$ of $50 \mathrm{mM}$ phosphate buffer ( $\mathrm{pH}$ 6.0) containing $0.167 \mathrm{mg} / \mathrm{ml} o$-dianisidine dihydrochloride (Sigma) and $0.005 \%$ hydrogen peroxide (Sigma). MPO activity was derived from an observed change in absorbance measured by spectrophotometry at $450 \mathrm{~nm}$ (Bio-Rad, California, USA) and normalized to the total protein content of the supernatant.

\section{Quantitative Real-time Polymerase Chain Reaction}

Total RNA was extracted from distal colonic specimens using the RNeasy Midi Kit (Qiagen). One microgram of total RNA was converted to single strand complementary DNA (cDNA) by reverse transcription (Superscript, Invitrogen) with oligo(dT) priming. One tenth of the cDNA was used in realtime quantification using the SYBR green kit (Eurogentec) and $250 \mathrm{nM}$ of each primer. A two-step program was run on the iCycler (Bio-Rad Laboratories). Cycling conditions were 
$95^{\circ} \mathrm{C}$ for $10 \mathrm{~min}, 40$ cycles of $95^{\circ} \mathrm{C}$ for $15 \mathrm{~s}$ and $60^{\circ} \mathrm{C}$ for $1 \mathrm{~min}$. Melting curve analysis confirmed primer specificities. All reactions were run in duplicate and normalized to hydroxymethyl-bilane synthase (HMBS) levels.

The efficiency of each primer pair was calculated using a standard curve of reference genomic DNA or cDNA (Roche). Amplification efficiency was determined using the formula $10^{-1 / \text { slope }}$. For the actual calculations, the base of the exponential amplification function was used (eg 2.04 in case of $104 \%$ amplification efficiency).

The primer pairs used for qPCR of the inflammatory cytokines TNF- $\alpha$, IL- 6 and IL- $1 \beta$ were published earlier. ${ }^{14}$ For mTNF- $\alpha$ - forward: catcttctcaaaattcgagtgacaa, reverse: tgggag tagacaaggtacaaccc; for mIL-6-forward: gaggataccactcccaacagacc, reverse: aagtgcatcatcgttgttcataca and for IL- $1 \beta$-forward: caaccaacaagtgatattctccatg, reverse: gatccacactctccag ctgca. The primer pair used for mHMBS was forward: aagggcttttctgaggcacc, reverse: agttgcccatctttcatcactg.

\section{Enzyme-Linked Immunosorbent Assay for VEGF and PLGF}

Snap-frozen distal colonic samples were immersed in $300 \mu \mathrm{l}$ of RadioImmunoPrecipitation Assay buffer before sonication on ice for $20 \mathrm{~s}$. Suspensions were separated by centrifugation at $16000 \mathrm{~g}$ for $20 \mathrm{~min}$. PlGF and VEGF protein levels were quantified by ELISA (Quantikine, R\&D, Minneapolis, USA) according to the manufacturer's instructions and normalized to the total protein content of the supernatant.

\section{CD31 Staining and Quantification of Mucosal Vascularization}

For CD31 staining, paraffin-embedded distal colonic sections were cut at a $5-\mu \mathrm{m}$ thickness, deparaffinized, hydrated and trypsinized for $7 \mathrm{~min}$ at $37^{\circ} \mathrm{C}$ using $12.5 \%$ trypsin (Sigma) in calcium chloride. Endogenous peroxidase was blocked with $0.3 \%$ hydrogen peroxide $\left(\mathrm{H}_{2} \mathrm{O}_{2}\right)$ in methanol. Incubation with the primary CD31 antibody (BD Pharmingen, California, USA) was carried out at a 1:500 dilution in TNB (0.1 M Tris-HCl, pH 7.5, 0.15 M NaCl) overnight at RT. A secondary antibody (rabbit anti-rat; Dako, California, USA) was used at a 1:300 dilution in TNB and applied for $45 \mathrm{~min}$. Detection was achieved using a commercialized streptavidinbiotin amplification system (Perkin Elmer) and antigen localization was visualized with 3'-3-diamino benzidene (DAB; Dako). Slight counterstaining was performed with hematoxyllin. Epitope specificity of the primary antibody was determined by treating consecutive sections with the same protocol, but replacing the primary antibody with an isotypespecific irrelevant antibody.

Computerized morphometric analysis was carried out as reported earlier using an international consensus method for quantification of angiogenesis with slight modifications. ${ }^{1,2}$ Briefly, CD31 immunostained sections were scanned at low magnification $(\times 40, \times 100)$ to detect the three most vascularized areas ('hot spots') in the mucosa, which was defined as the area above the muscularis mucosae. Pictures of those areas were taken at high magnification $(\times 400)$ using an Optronics Color digital camera (Olympus Corporation, Tokyo, Japan). The picture was oriented to permit counting of the maximum number of vessels per high-power field. The number of vessels/field (the mean vascular density, MVD) and the mean vessel diameters were obtained by quantitative analysis using specialized software (Cell D, Olympus Soft Imaging Solutions, Münster, Germany). The means of all measurements per section were used for analysis.

\section{Intestinal Epithelial Apoptosis Detection}

Sections of the distal colonic tissue were stained using the terminal deoxynucleotidyl transferase (TdT) dUTP-nick-end labeling (TUNEL) method using a commercial apoptosis detection kit (Genscript, New Jersey, USA). Briefly, paraffinembedded distal colonic sections were cut at a $5-\mu \mathrm{m}$ thickness, deparaffinized, hydrated and incubated with a proteinase $\mathrm{K}$ for $25 \mathrm{~min}$ and blocked with $3 \% \mathrm{H}_{2} \mathrm{O}_{2}$ in methanol for $10 \mathrm{~min}$. After rinsing three times with PBS, a freshly prepared TUNEL reaction mixture containing 92\% Equilibration Buffer, 2\% biotin-11-dUTP and 6\% TdT was added to the samples for $1 \mathrm{~h}$ at $37^{\circ} \mathrm{C}$. After rinsing, samples were incubated with a Streptavidin-HRP Solution for $30 \mathrm{~min}$ at $37^{\circ} \mathrm{C}$. Detection was performed with $\mathrm{DAB}$ and slides were counterstained with hematoxyllin. Negative control samples underwent the same process with omission of the TdT enzyme. Positive control samples were pre-treated with $100 \mathrm{U} / \mathrm{ml}$ DNase I for $10 \mathrm{~min}$ at $15-25^{\circ} \mathrm{C}$ to induce DNA strand degradation before the labeling procedure.

Distal colonic epithelial apoptosis was blindly scored assessed by two independent observers (PH and DL) using the following scoring system: 0 (baseline, no detection or single apoptotic cells); 1 (mildly increased apoptosis with clearly defined apoptotic areas in $<5 \%$ of the surface epithelium); 2 (moderately increased apoptosis with clearly defined apoptotic areas in 5-20\% of surface epithelium) and 3 (severely increased apoptosis with clearly defined apoptotic areas in $>20 \%$ of surface epithelium).

\section{Pimonidazole Staining}

To show early DSS-induced colonic hypoxia, $\mathrm{PlGF}^{-1-}$ mice $(N=3)$ and $\mathrm{PlGF}^{+1+}$ mice $(N=3)$ were treated for 3 days with DSS and injected intraperitoneally with $60 \mathrm{mg} / \mathrm{kg}$ of pimonidazole $1 \mathrm{~h}$ before killing. Pimonidazole rapidly diffuses out of the cells in the presence of normal oxygen levels. In the case of inadequate oxygen concentrations, the molecule is retained. ${ }^{15}$ Following the manufacturer's instructions, distal colonic pimonidazole distribution was visualized on paraffin-embedded sections using a hypoxyprobe-plus kit (Natural Pharmacia International Inc, Massachusetts, USA) and compared with baseline conditions (healthy controls: two $\mathrm{PlGF}^{+/+}$, two $\mathrm{PlGF}^{-/-}$). 


\section{Statistical Analysis}

The data were analyzed using SPSS version 16.0 for Windows (SPSS Inc, Illinois, USA).

Variables are reported as the mean \pm standard error of the mean (s.e.m.) or as the median \pm range. In the case of normal data distribution, groups were compared by using Student's $t$-test for independent samples. For non-normal or unknown data distribution, a Mann-Whitney $U$-test was performed. For histological inflammation and apoptosis detection, the mean of the two independent observers' scores was considered for analysis. Clinical disease activity scores of $\mathrm{PlGF}^{+/+}$and $\mathrm{PlGF}^{-1-}$ mice were compared by ANOVA for repeated measurements.

Two-tailed probabilities were calculated and results were considered significant at a value of $P<0.05$.

\section{RESULTS}

\section{Both VEGF and PLGF Are Involved in Acute Colitis}

We first assessed the involvement of PIGF vs VEGF in DSS colitis by analyzing the level of VEGF and PIGF in distal colonic lysates at several time points of disease. Both VEGF and PlGF are upregulated during acute DSS colitis. A significant increase in PIGF concentration was observed in the $\mathrm{PlGF}^{+/+}$mice as early as 3 days after DSS administration (the fold increase at day 3 compared with baseline at day 0 was $2.62 \pm 0.35, P<0.05)$. A further increase in PlGF was also seen on days 7 and $11(5.06 \pm 2.83$ and $6.24 \pm 1.6$, respectively) (Figure 1a).
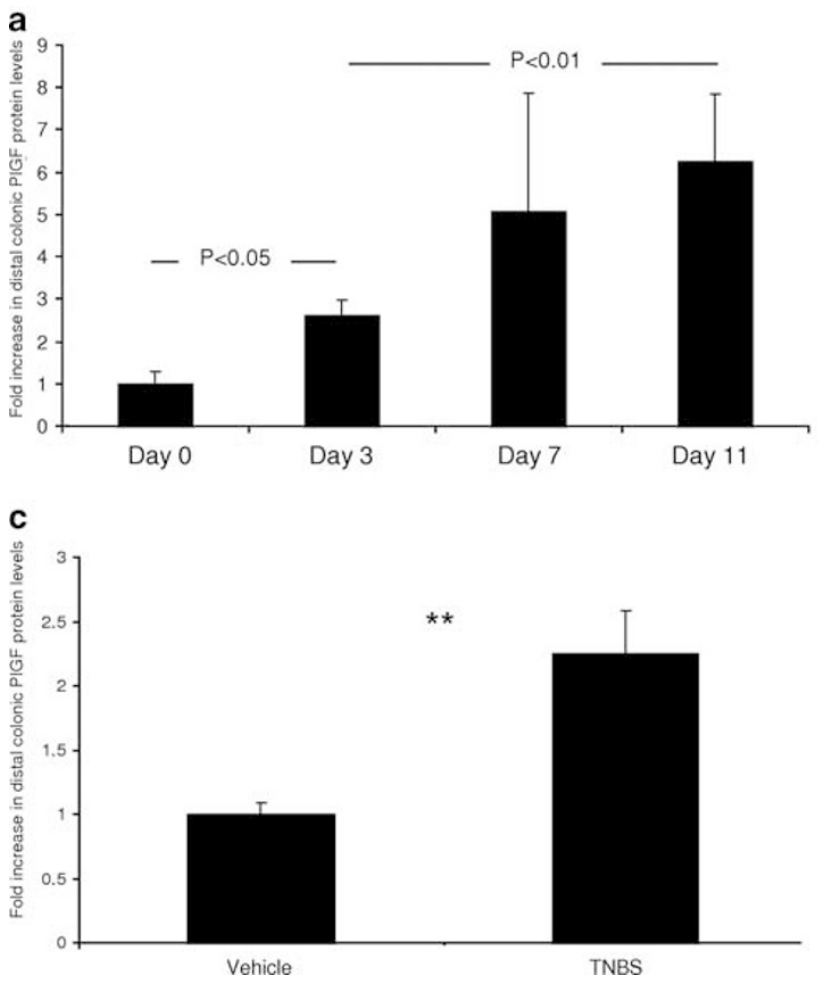

Changes in VEGF concentration in the $\mathrm{PlGF}^{+1+}$ mice treated with DSS were less pronounced and observed later than the changes in PlGF (Figure 1b). A significant increase in VEGF concentration was seen on day 11 (the fold increase at day 11 compared with baseline at day 0 was $1.54 \pm 0.15$, $P<0.05)$. In contrast, distal colonic VEGF activity in $\mathrm{PlGF}^{-1-}$ mice was significantly enhanced at day 7 (a fold increase of $1.43 \pm 0.14, P<0.05$ ). The increase was more pronounced in $\mathrm{PlGF}^{-1-}$ compared with the $\mathrm{PlGF}^{+/+}$controls (fold change at day 7 of $1.43 \pm 0.14$ and $1.07 \pm 0.24$, respectively; $P<0.05$; Figure 1b).

In addition, in TNBS colitis, a significant increase in colonic PlGF protein levels was seen (fold increase at day 3 compared with baseline at day 0: $2.25 \pm 0.34, P<0.05$; Figure 1c), whereas the VEGF protein levels were not significantly altered (Figure 1d).

\section{PIGF $^{-1-}$ Mice Show Increased Disease Activity during Acute Colitis}

During the course of the DSS colitis, significant differences in weight loss (Figure 2a) and rectal bleeding were seen between the $\mathrm{PlGF}^{-1-}$ and the $\mathrm{PlGF}^{+/+}$group. These symptoms of disease indicate a significantly higher disease activity index in $\mathrm{PlGF}^{-1-}$ mice (compared with $\mathrm{PlGF}^{+1+}$ mice: ANOVA; $P<0.001)$.

DSS treatment resulted in significant shortening of the colon length both in $\mathrm{PlGF}^{+1+}$ and $\mathrm{PlGF}^{-1-}$ mice (Figure 2b). However, shortening was enhanced in the $\mathrm{PlGF}^{-1-}$
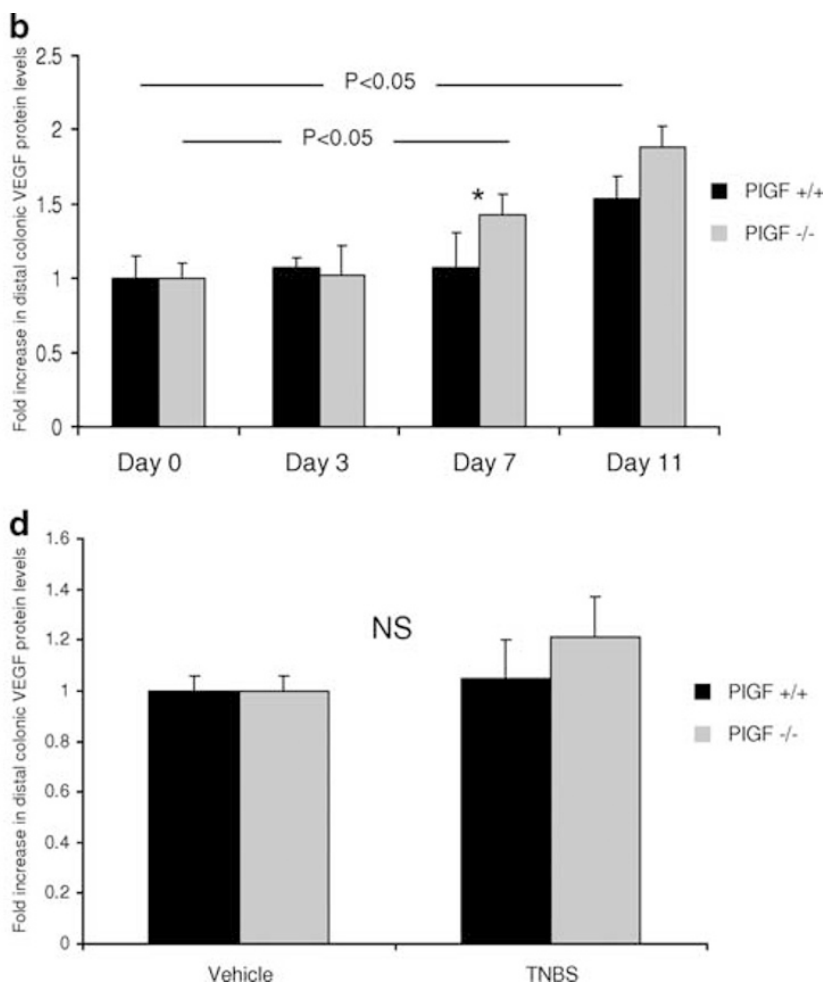

Figure $1(\mathbf{a}, \mathbf{b})$ Distal colonic levels of PIGF $(\mathbf{a})$ and VEGF $(\mathbf{b})$ protein in $\mathrm{PIGF}^{+/+}$vs $\mathrm{PIGF}^{-/-}$mice after DSS administration. (c, $\left.\mathbf{d}\right)$ Distal colonic levels of PIGF (c) and VEGF (d) protein in PIGF ${ }^{+/+}$vs $\mathrm{PIGF}^{-/-}$mice after TNBS administration. ${ }^{\star} P<0.05,{ }^{* *} P<0.01$. 

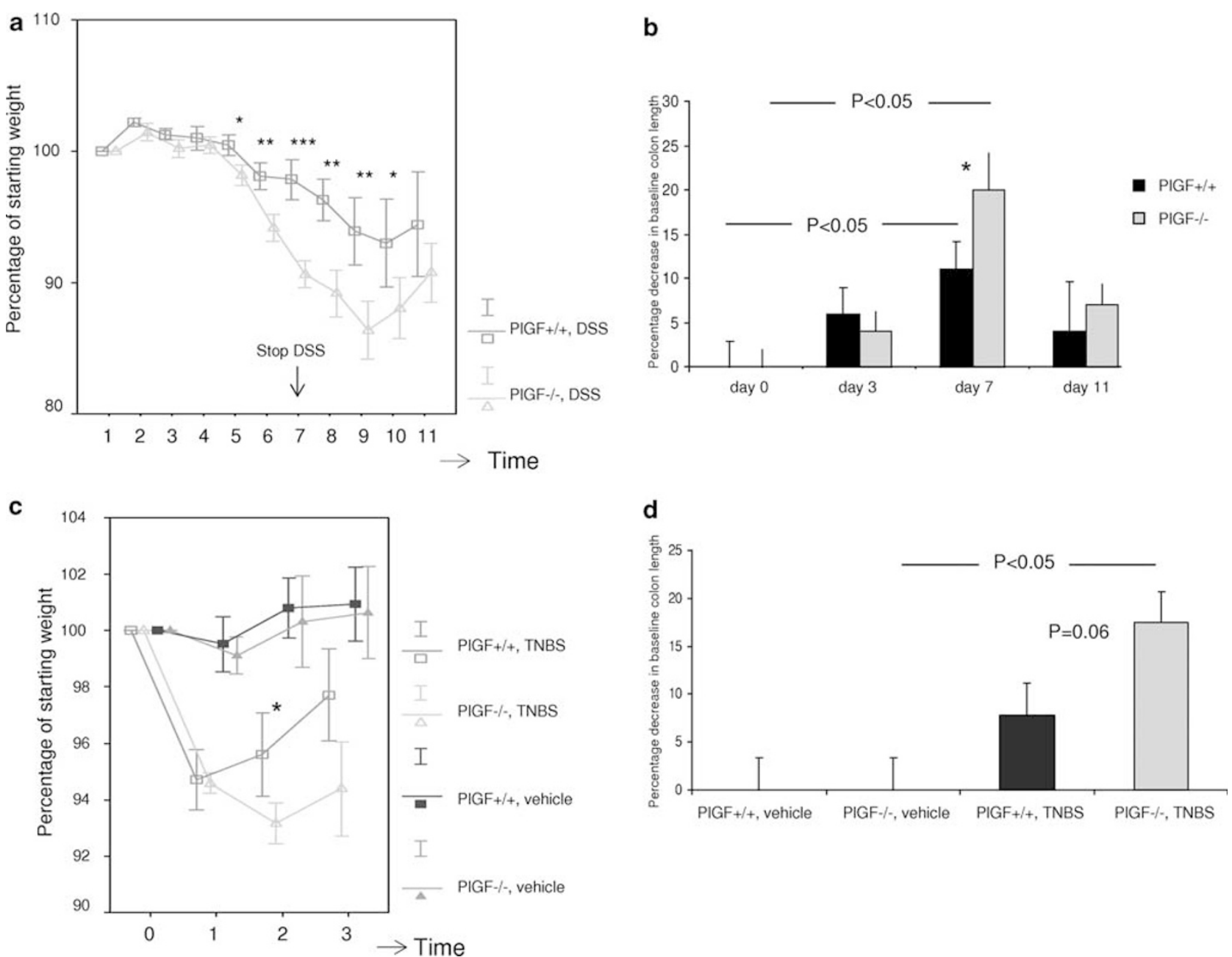

Figure 2 Weight evolution after DSS (a) and TNBS (c) administration in $\mathrm{PIGF}^{+/+}$vs $\mathrm{PIGF}^{-1-}$ mice. Bars present mean \pm s.e.m. ${ }^{\star} P<0.05,{ }^{* *} P<0.01$, ${ }_{* * * P}<0.001$. Percent decrease in colon length in $\mathrm{PIGF}^{+/+}$vs $\mathrm{PIGF}^{-/-}$mice after DSS (b) and TNBS (d) administration.

group compared with the $\mathrm{PlGF}^{+1+}$ group (the percentage decrease in colon length on day 7 was $20 \pm 4.2$ and $11 \pm 3.1$, respectively; $P<0.05$ ).

To better define the function of PlGF in acute colitis, we investigated the effects of PlGF deletion in TNBS-induced colitis as well. In addition, in this model, the absence of PlGF was associated with significantly aggravated disease course, objectivated by increased weight loss (compared with $\mathrm{PlGF}^{+/+}$ mice: ANOVA; $P<0.01)$ and a nearly significant increase in colonic shortening compared with $\mathrm{PlGF}^{+1+}$ mice $(P=0.06)$ (Figure $2 \mathrm{c}$ and $\mathrm{d}$ ). The similar results obtained in both models establish the importance of PlGF in acute colitis.

\section{Poor Outcome in $\mathrm{PIGF}^{-1-}$ Mice during Acute DSS- induced Colitis Is Associated with Increased Intestinal Epithelial Damage, Which Is Not Caused by Hyperinfiltration of Leukocytes}

Next, we looked at the histopathological correlation of the increased DSS-induced disease activity in $\mathrm{PlGF}^{-1-}$ mice. Inter-observer agreement for histological score on H\&E- stained sections was excellent $(\kappa=0.89)$. No significant increase in inflammation score was noted in $\mathrm{PlGF}^{-1-}$ compared with $\mathrm{PlGF}^{+1+}$ mice. Consistent with this, there were no significant differences in MPO levels between the two groups (Figure $3 \mathrm{a}$ and $\mathrm{b}$ ). In contrast, obvious differences were found at the level of colonic epithelial damage. After 7 days of DSS treatment, the epithelial damage score was significantly $(P<0.05)$ higher in $\mathrm{PlGF}^{-1-}$ (median 0 , range 0-2.5) compared with $\mathrm{PlGF}^{+/+}$mice (median 2.5, range $0-7$ ) (Figure 3c, illustrations Figure 3d).

Both colonic TNF- $\alpha$ and IL- 6 production were significantly increased in $\mathrm{PlGF}^{-1-}$ mice $v s \mathrm{PlGF}^{+/+}$mice at day 7 of DSS colitis (fold increase compared with baseline: for TNF- $\alpha$ : $12.01 \pm 3.20$ for $\mathrm{PlGF}^{+/+}$mice $v s 23.79 \pm 1.52$ for $\mathrm{PlGF}^{-1-}$ mice; $P<0.05$ and for IL-6: $2.89 \pm 0.66$ for $\mathrm{PlGF}^{+/+}$mice $v s$ $17.53 \pm 3.09$ for PlGF ${ }^{-1-}$ mice; $P<0.01$ ) (Figure 3e). Colonic production of IL-1 $\beta$ did not significantly differ between both groups (data not shown).

To investigate whether the increase in histological damage in $\mathrm{PlGF}^{-1-}$ mice was preceded by enhanced intestinal 


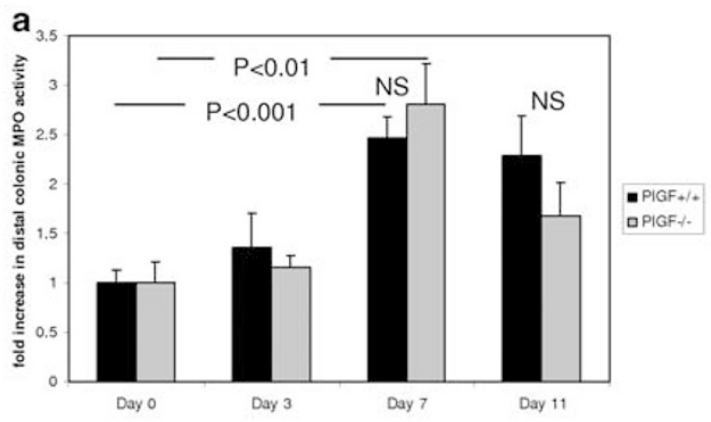

b
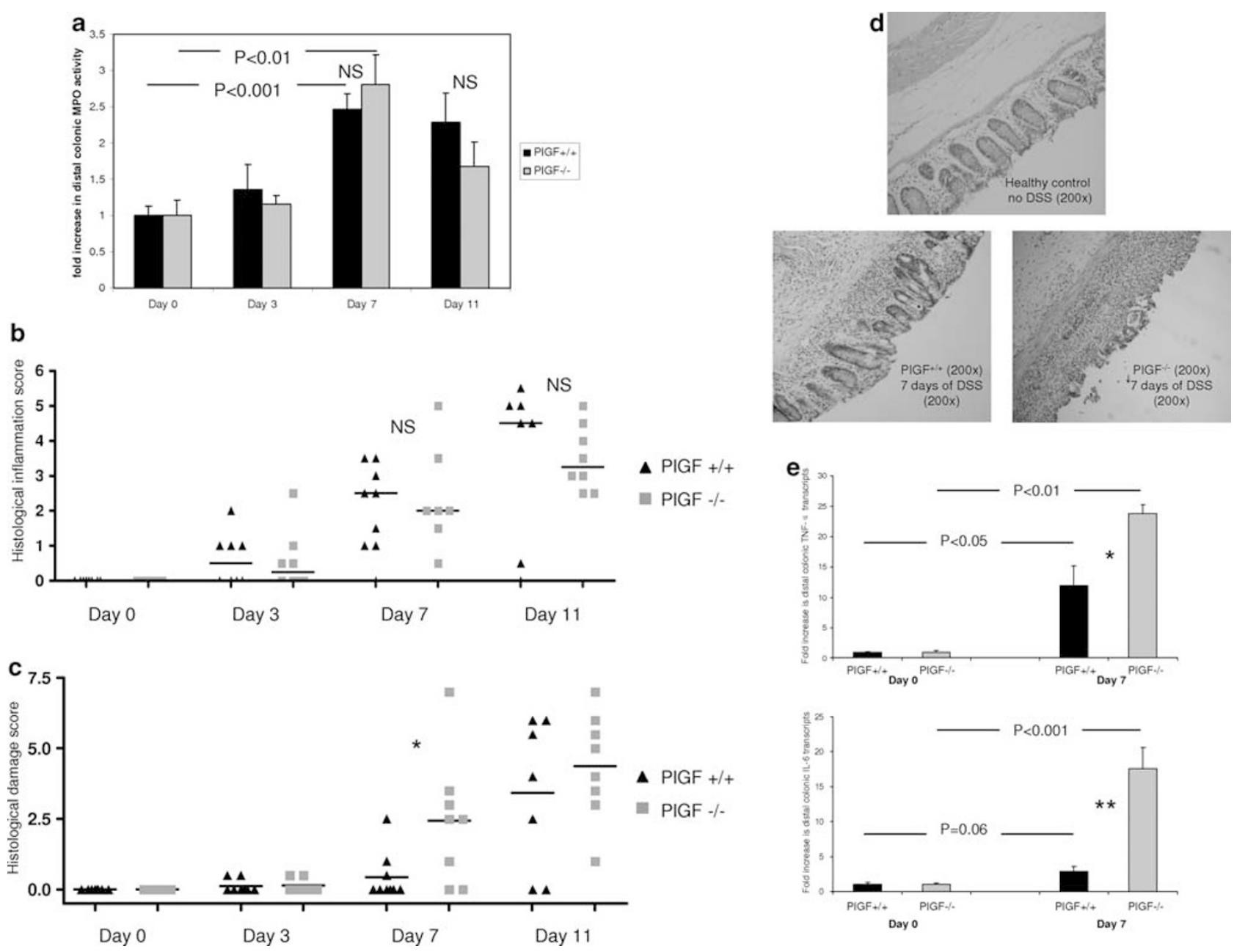

Figure 3 (a) Distal colonic MPO-levels during DSS colitis in $\mathrm{PIGF}^{+/+}$and $\mathrm{PIGF}^{-/-}$mice. (b) Histological inflammation score during DSS colitis in PIGF ${ }^{+/+}$ and PIGF ${ }^{-1-}$ mice. (c) Histological damage score during DSS colitis in $\mathrm{PIGF}^{+/+}$and PIGF ${ }^{-1-}$ mice. (d) Representative pictures of H\&E-stained distal colonic sections. Note the increased crypt damage and ulcerations in DSS-treated PIGF ${ }^{-1-}$ mice (lower right picture) compared with DSS-treated PIGF ${ }^{+/+}$mice (lower left picture). (e) Fold increase in distal colonic TNF- $\alpha$ and II-6 mRNA levels during DSS colitis in PIGF ${ }^{+1+} v$ PIGF $^{-1-}$ mice. $^{\star} P<0.05,{ }^{* *} P<0.01$.

epithelial apoptosis, we performed a TUNEL assay. Using our apoptosis scoring system, inter-observer agreement was moderate $(\kappa=0.57)$. However, there were no unacceptable discrepancies (change in score of observer 1 vs score of observer $2>1$ ) between the observers. Consistent with existing literature, increased epithelial apoptosis was observed early (day 3 after DSS administration) and before the appearance of clear histological inflammation or epithelial ulcerations. ${ }^{16-19}$ However, epithelial apoptosis was significantly $(P<0.05)$ more pronounced in DSS-treated $\mathrm{PlGF}^{-1-}$ mice (median 0.5, range 0-3) compared with $\mathrm{PLGF}^{+/+}$mice (median 0, range 0-1.5) (Figure 4).

\section{Increased Disease Activity of PIGF ${ }^{-1-}$ Mice during Acute DSS-Induced Colitis Is Preventable by Exogeneous Administration of Recombinant PIGF}

To fully confirm the protective function of PlGF during acute colitis, we investigated whether exogeneous adminis- tration of PlGF by adenoviral transfection could reverse the bad outcome of $\mathrm{PlGF}^{-/-}$mice after DSS administration. An IV injection of $1 \times 10^{9}$ pfu AdPlGF led to a stable increase of distal colonic PlGF levels, in the expected range of PlGF $+1+$ mice after a 7-day administration of DSS (Figure 5a). In contrast to AdRR5-treated $\mathrm{PlGF}^{-1-}$ mice, distal colonic VEGF levels of AdPlGF-treated $\mathrm{PlGF}^{-1-}$ mice were not significantly enhanced compared with $\mathrm{PlGF}^{+/+}$mice (Figure 5b). AdPlGF-treated mice did significantly better during DSS colitis as compared with AdRR5-treated mice (ANOVA: $P<0.001$ ). In contrast to AdRR5-treated $\mathrm{PlGF}^{-1-}$ mice, no significant difference in weight loss was observed in AdPlGF-treated mice as compared with $\mathrm{PlGF}^{+1+}$ mice (Figure 5c). In accordance, the histological damage score was significantly lower in AdPlGF-treated $\mathrm{PlGF}^{-1-}$ mice compared with AdRR5-treated $\mathrm{PlGF}^{-1-}$ mice (median 2, range $0-5$ vs median 5 , range $3-5$, respectively; $P<0.05$ ) (Figure 5d). 


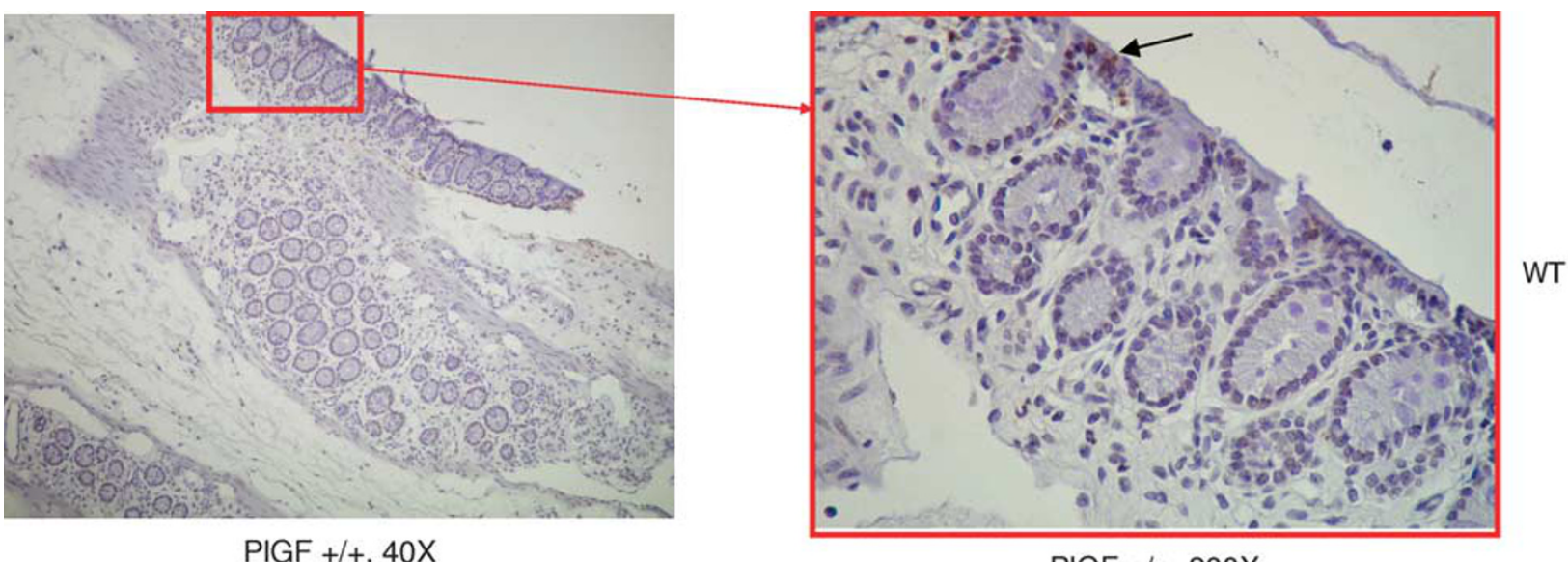

PIGF +/+, 200X

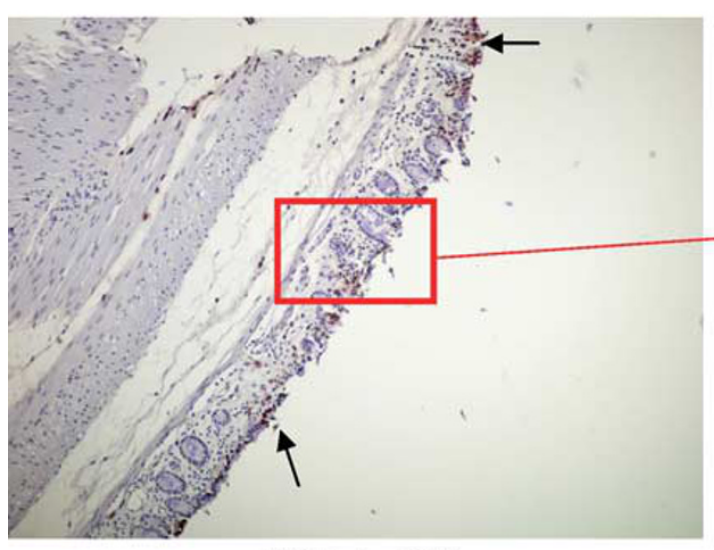

PIGF - /- , 40X

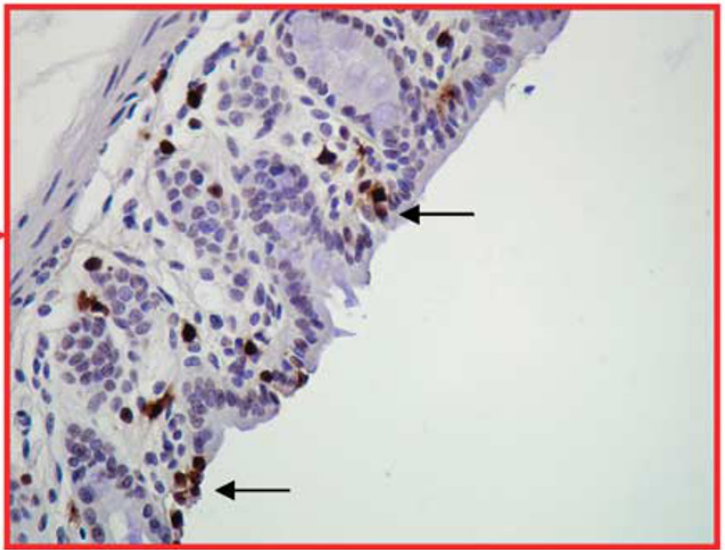

PIGF -/- , 200X

Figure 4 Representative images $\left(\times 40\right.$ and $\times 200$ ) of TUNEL-stained distal colonic sections from PIGF ${ }^{+/+}$and PIGF ${ }^{-/-}$mice after 3 days of DSS treatment. Apoptotic cells possess dark brown-stained nuclei (some of them are marked with black arrows). Note the increased epithelial apoptosis in PIGF ${ }^{-/-}$mice $^{-}$ compared with $\mathrm{PIGF}^{+/+}$mice.

\section{Loss of PIGF Blocks Colonic Mucosal Angiogenesis During DSS-Induced Colitis}

Next, we determined whether PIGF is important in DSS-induced angiogenesis by analyzing CD31 staining in distal colonic sections. At baseline, no obvious differences in intestinal mucosal vascularization were seen between $\mathrm{PlGF}^{-1-}$ and $\mathrm{PlGF}^{+1+}$ mice. However, mucosal angiogenic activity during DSS-induced colitis was strikingly different (Figure 6). As early as 3 days after DSS administration, $\mathrm{PlGF}^{+1+}$ mice displayed an increase both in MVD, the number of blood vessels/high-power field (Figure 6a) and mean vessel diameter (Figure 6b). The differences in vascularization were significant on day 7 (a fold increase of $1.59 \pm 0.14, P<0.05$ for MVD and $1.44 \pm 0.12, P<0.05$ for mean vessel diameter). In contrast to $\mathrm{PlGF}^{+/+}$mice, $\mathrm{PlGF}^{-1-}$ mice showed no significant changes in either mucosal vascularization or mean vessel diameter after DSS administration (Figure 6c).

\section{Evidence for Early Enhanced Colonic Epithelial Hypoxia in DSS-Treated PIGF $^{-l-}$ Mice}

It is known from literature that acute experimental colitis is associated with a significant increase in colonic mucosal hypoxia and that colonocytes are the primary targets of this oxygen depletion. ${ }^{15,20}$

As the early increase in colonocyte apoptosis in $\mathrm{PlGF}^{-1-}$ mice was associated with an early decrease in DSS-induced mucosal angiogenesis, we hypothesized that rapidly enhanced intestinal epithelial hypoxia may cause the differences observed between $\mathrm{PlGF}^{-1-}$ and $\mathrm{PlGF}^{+/+}$mice. To investigate this, we injected three $\mathrm{PlGF}^{+/+}$and three $\mathrm{PlGF}^{-1-}$ mice with pimonidazole after treating the mice with DSS for 3 days (a time point we had observed clear differences in colonic mucosal vascularization and colonocyte apoptosis between the $\mathrm{PlGF}^{+1+}$ and $\mathrm{PlGF}^{-1-}$ mice). Pimonidazole tissue distribution in these mice was compared with healthy controls $(N=4)$. Consistent with earlier findings, physiologic hypoxia in the colonic surface epithelium was observed under baseline conditions in both $\mathrm{PlGF}^{+/+}$and $\mathrm{PlGF}^{-/-}$mice. ${ }^{19}$

After 3 days of DSS treatment, $\mathrm{PlGF}^{+1+}$ mice did not show any sign of increased pimonidazole uptake compared with healthy controls (Figure 7). On the contrary, clear increases in colonic epithelial pimonidazole uptake extending to the deeper parts of the crypts were observed in $\mathrm{PlGF}^{-1-}$ 

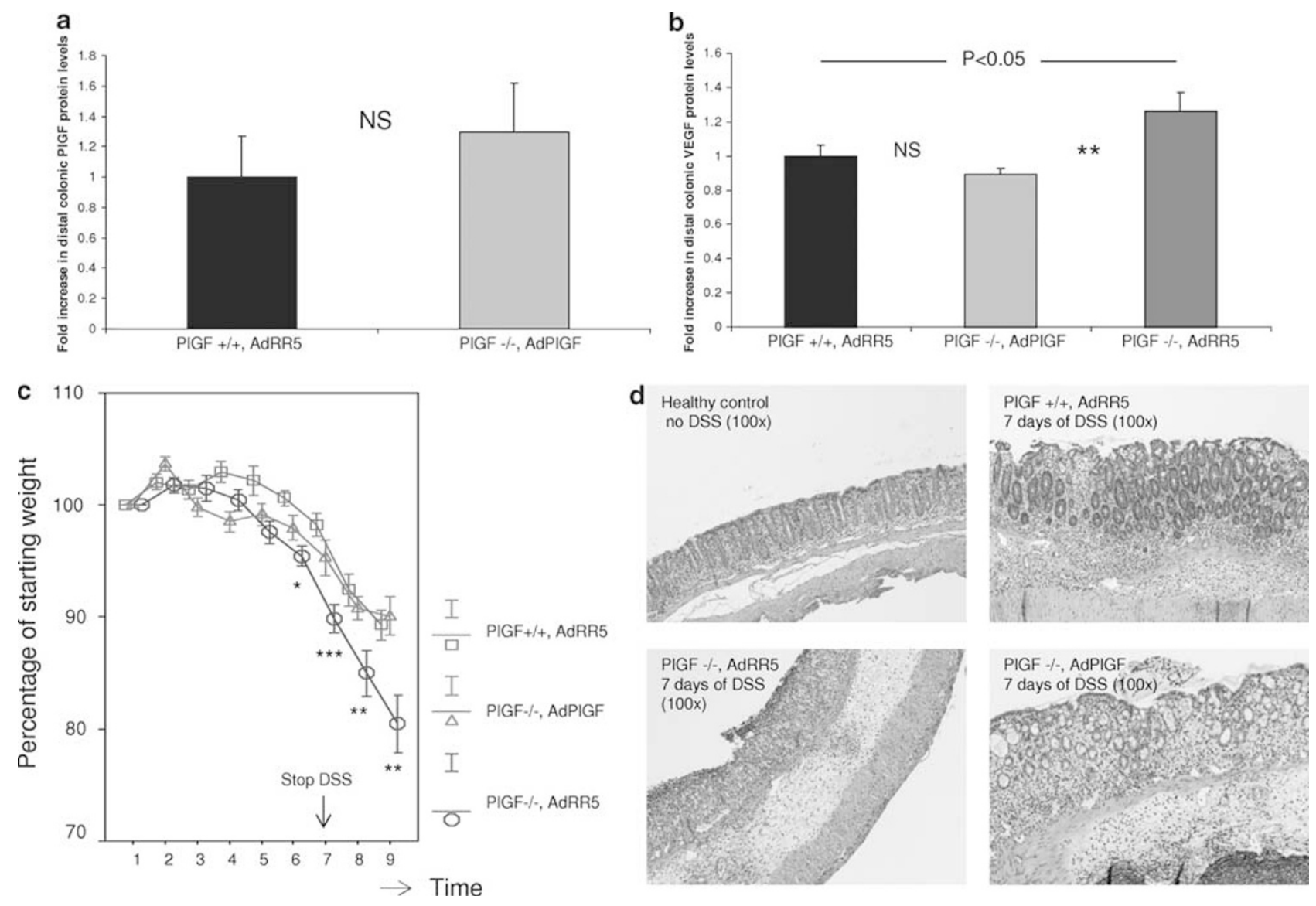

Figure 5 Distal colonic protein levels of PIGF (a) and VEGF (b) during DSS colitis (day 9) in PIGF ${ }^{-1-}$ mice treated with a PIGF-overproducing adenovirus (AdPIGF) and PIGF ${ }^{+/+}$mice treated with a control adenovirus (AdRR5). PIGF-levels in AdRR5-treated PIGF ${ }^{-1-}$ mice were zero and are not shown. (c) Weight evolution after DSS administration in AdPIGF-treated PIGF ${ }^{-/}$mice and AdRR5-treated PIGF ${ }^{-1-}$ and PIGF ${ }^{+/+}$mice. Bars present mean \pm s.e.m. AdPIGFtreated $\mathrm{PIGF}^{-1-}$ mice vs AdRR5-treated $\mathrm{PIGF}^{-1-}$ mice: ${ }^{*} P<0.05,{ }^{* *} P<0.01,{ }^{* *} P<0.001$. (d) Representative pictures of H\&E-stained distal colonic sections. AdPIGF-treated PIGF ${ }^{-1-}$ mice (lower right picture) showed less severe crypt destruction and ulcerations after DSS administration as compared with AdRR5-treated $\mathrm{PIGF}^{-1-}$ mice (lower left picture).

mice. These data indicate an enhanced epithelial hypoxia in $\mathrm{PlGF}^{-1-}$ mice.

\section{DISCUSSION}

In this study, we report for the first time that intestinal mucosal angiogenesis in experimental colitis is critically dependent on PlGF. The absence of PlGF expression was found to worsen the disease course in two different models of acute colitis by aggravating colonic epithelial damage. We provide evidence for a function of early mucosal hypoxia enhancement as the underlying mechanism. Owing to their anatomical position, colonocytes are particularly susceptible to insufficient blood and oxygen supply. ${ }^{19}$ The resulting mucosal hypoxia may render the colonocytes apoptotic, resulting in a disruption of the epithelial barrier. ${ }^{21,22} \mathrm{PlGF}^{-1-}$ mice have been shown earlier to possess a reduced ability to respond to ischemic damage compared with $\mathrm{PlGF}^{+/+}$mice. ${ }^{4}$ Moreover, PlGF seems to have beneficial effects in a number of hypoxia-associated conditions. ${ }^{6,8}$
$\mathrm{PlGF}^{-1-}$ mice showed a more pronounced increase in VEGF protein levels in the distal colon after DSS administration compared with $\mathrm{PlGF}^{+/+}$mice, which was preventable by exogeneous administration of PlGF. Interestingly, this boost in VEGF was not able to compensate for the decrease in angiogenesis caused by the absence of PIGF. A similar finding has been described in the ischemic retina. $^{4}$

Several potential mechanisms by which PlGF and VEGF exert different effects have been proposed. VEGF both binds VEGF receptor-1 (FLT1) and VEGF receptor-2 (FLK1), whereas PlGF only binds FLT1. As such, an increase in PlGF may displace VEGF from FLT1 and thereby increase the fraction of VEGF available to activate its main receptor, FLK1. Alternatively, crosstalking of FLT1 and FLK1 after binding of PlGF to FLT1 is able to amplify VEGF signaling through FLK1. Finally, PIGF is able to induce its own angiogenic signal through FLT1 independently of VEGF. ${ }^{4,23}$ Synergy between PlGF and VEGF in pathological angiogenesis in the gut may have important therapeutic implications 


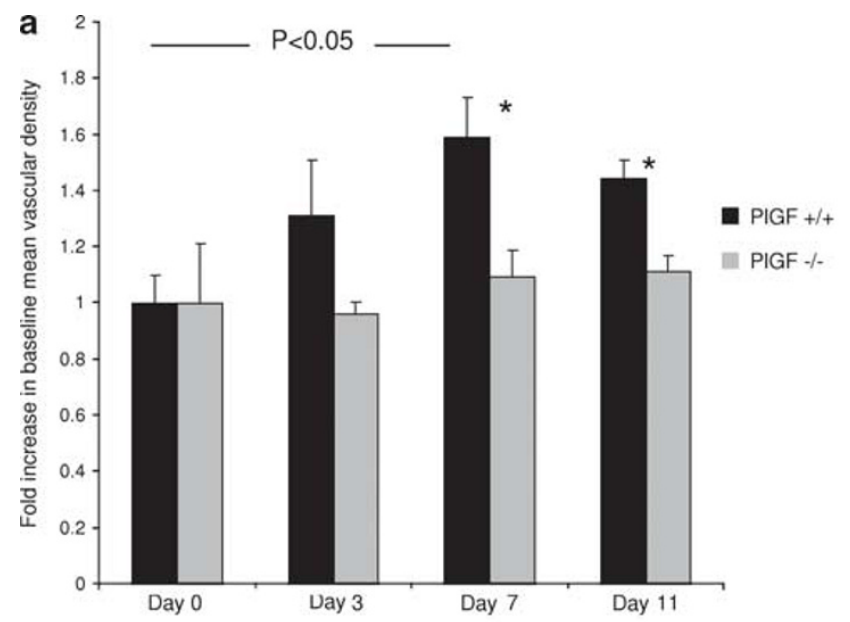

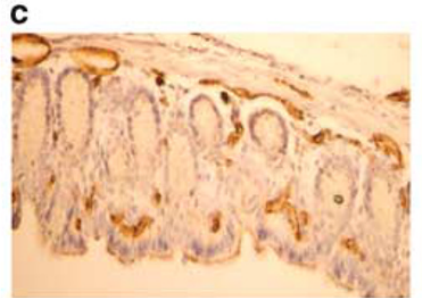

Distal colon (100x), PIGF $+1+$ control

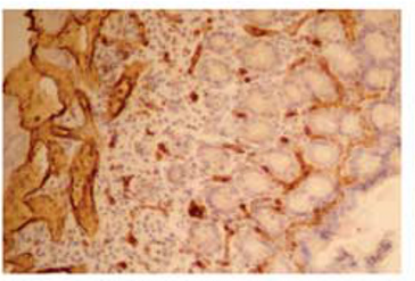

Distal colon (100x), PIGF $+/+7$ days of DSS Distal colon (100x), PIGF $\% 7$ days of DSS

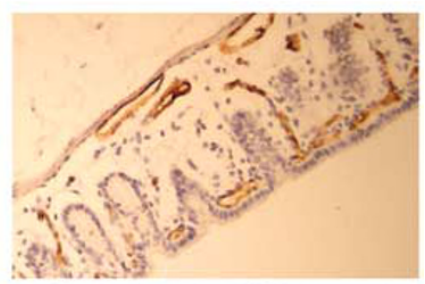

Distal colon (100x), PIGF $\%$ control

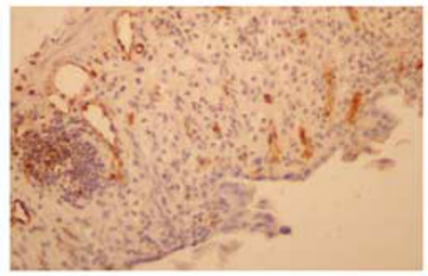

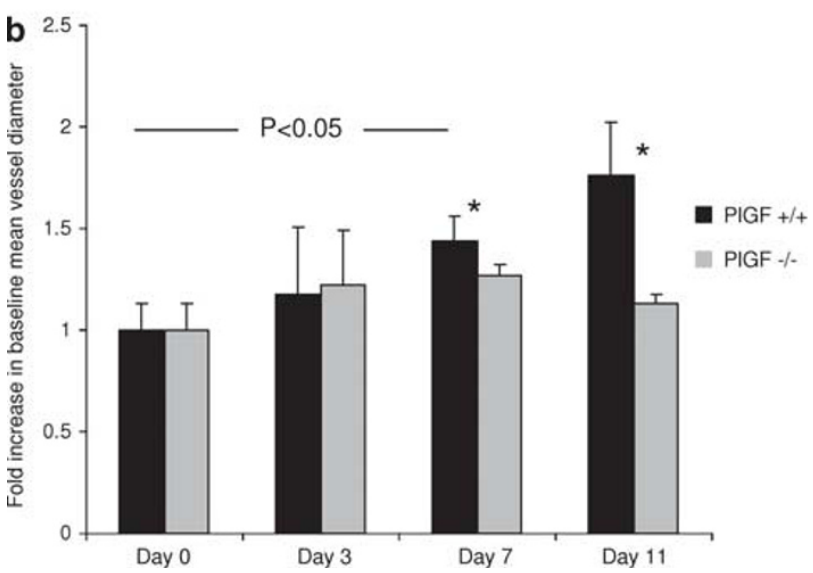

Figure 6 Mean vascular density (MVD, a) and mean vessel diameter (b) in PIGF ${ }^{+/+}$vs PIGF $^{-/-}$mice after DSS administration. (c) Representative images of CD31-stained distal colonic sections $(\times 100)$. Of note, the lack of DSS-induced angiogenesis in PIGF ${ }^{-1-}$ mice (lower right picture) is associated with severe colonic crypt destruction as compared with the $\mathrm{PIGF}^{+/+}$controls (lower left picture). ${ }^{\star} P<0.05$.

for either stimulating or inhibiting angiogenesis in patients with IBD.

To our knowledge, only two papers provide data on PlGF expression in patients with IBD. Kader et $a l^{24}$ measured the serum PlGF concentration in 88 pediatric patients with confirmed IBD. They determined that PIGF was higher in patients in remission $v s$ patients with active disease. Pousa et $a l^{25}$ measured serum PlGF levels in 70 adult patients with confirmed Crohn's disease in clinical remission and found the level of PIGF to be significantly increased compared with healthy controls.

Our findings initially seem to contradict some, but not all earlier reports on angiogenesis in experimental models of colitis. Danese et $a l^{2}$ found that the anti-angiogenic compound ATN-161, a dual AV $\beta 3 / 5 \beta 1$ inhibitor, has convincing therapeutic effects in the chronic $\mathrm{IL}_{10} 0^{-/}$colitis model. The same benefit was seen by Chidlow et $a^{26}$ in the CD4 + CD45RBhigh model. However, ATN-161 had no therapeutic action in DSS-induced colitis. ${ }^{2}$ An explanation for the differences in angiogenesis between these colitis models may be found in the nature of DSS-induced colitis.
The observed inflammation in DSS-induced colitis is likely a secondary response after induction of non-inflammatory epithelial damage. ${ }^{10,27}$ Therefore, the DSS model may in fact be seen as an intestinal epithelial wound-healing model. This situation is very different from murine models that spontaneously develop colitis (such as $\mathrm{IL}-10^{-/-}$and CD4 + CD45RBhigh mice), in which an inflammatory insult is the primary cause of the colitis. ${ }^{28}$ In these models, angiogenesis may feed the disease process by facilitating the influx of inflammatory cells, whereas in the DSS-colitis model, angiogenesis would be required for proper wound healing and resolution of the injury. Therefore, we strongly believe that it is important to apply pro- or anti-angiogenic agents in a timely manner and target the correct angiogenic factor to beneficially influence the disease course of IBD.

In addition to its angiogenic capacity, PlGF has another biological activity, which may be relevant in DSS-induced colitis. PlGF strongly recruits and activates VEGFR-1 expressing macrophages. ${ }^{4,5}$ Recently, it has been shown that resident intestinal macrophages serve a protective function during the development of acute DSS-induced colitis. 


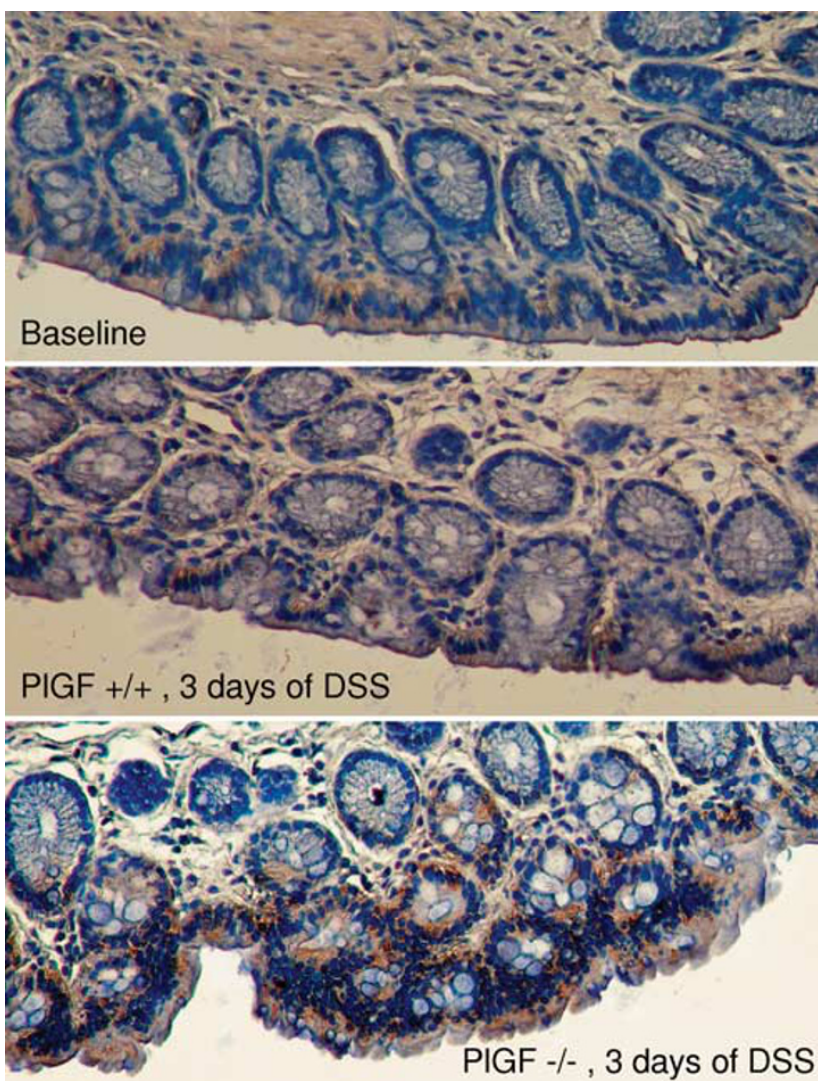

Figure 7 Representative images $(\times 200$ ) of pimonidazole-stained distal colonic sections from $\mathrm{PIGF}^{+/+}$and $\mathrm{PIGF}^{-1-}$ mice, baseline and after 3 days of DSS treatment. Note the early enhanced mucosal epithelial hypoxia in $\mathrm{PIGF}^{-1-}$ mice at day 3 of DSS treatment.

Intestinal macrophages facilitate phagocytosis of DSS and bacteria, regulate neutrophil migration and produce growth factors, which stimulate tissue repair. ${ }^{29}$ As sections stained with the murine pan macrophage marker F4/80 revealed no clear differences in baseline macrophage numbers between $\mathrm{PlGF}^{+/+}$and $\mathrm{PlGF}^{-1-}$ mice (data not shown), we did not further investigate this possibility.

In summary, our data show that the VEGF-homologue, PlGF, has an important function in two different murine models of colitis and in the angiogenesis associated with colitis. Loss of PlGF is linked with a worse clinical outcome in both models. Taken together, these data indicate that before targeting angiogenesis in a condition hallmarked by tissue destruction and repair, such as IBD, we need to know more about the exact function of this observed neovascularization on disease onset and maintenance.

\section{ACKNOWLEDGEMENTS}

We thank Kim Olievier for her assistance with the experiments. This study was supported by a concerted grant GOA2001/12051501 from Ghent University, Belgium, and by a grant of the National Fund for Scientific Research (FWO grant A2/5-11716). The work of PC is supported by longterm structural funding-Methusalem Funding by the Flemish Government,
Interuniversity Attraction Poles Belgian Government—IUAP P6/30 and concerted Grant GOA2006/11 from KU Leuven.

\section{DISCLOSURE/CONFLICT OF INTEREST}

The authors declare no conflict of interest.

1. Danese $S$, Sans $M$, de la Motte $C$, et al. Angiogenesis as a novel component of inflammatory bowel disease pathogenesis. Gastroenterology 2006;130:2060-2073.

2. Danese $S$, Sans $M$, Spencer DM, et al. Angiogenesis blockade as a new therapeutic approach to experimental colitis. Gut 2007;56:855-862.

3. Pousa ID, Maté J, Gisbert JP. Angiogenesis in inflammatory bowel disease. Eur J Clin Invest 2008;38:73-81.

4. Carmeliet $P$, Moons $L$, Luttun $A$, et al. Synergism between vascular endothelial growth factor and placental growth factor contributes to angiogenesis and plasma extravasation in pathological conditions. Nat Med 2001;7:575-583.

5. Fischer C, Jonckx B, Mazzone $M$, et al. Anti-PIGF inhibits growth of VEGF(R)-inhibitor-resistant tumors without affecting healthy vessels. Cell 2007;131:463-475.

6. Luttun $A$, Tjwa $M$, Moons $L$, et al. Revascularization of ischemic tissues by PIGF treatment, and inhibition of tumor angiogenesis, arthritis and atherosclerosis by anti-Flt1. Nat Med 2002;8:831-840.

7. Cianfarani $F$, Zambruno G, Brogelli $L$, et al. Placental growth factor in diabetic wound healing: altered expression and therapeutic potential. Am J Pathol 2006;169:1167-1182.

8. Kolakowski Jr S, Berry MF, Atluri $\mathrm{P}$, et al. Placental growth factor provides a novel local angiogenic therapy for ischemic cardiomyopathy. J Card Surg 2006;21:559-564.

9. Okayasu I, Hatakeyama $S$, Yamada $M$, et al. A novel method in the induction of reliable experimental acute and chronic ulcerative colitis in mice. Gastroenterology 1990;98:694-702.

10. Heymans $S$, Luttun $A$, Nuyens $D$, et al. Inhibition of plasminogen activators or matrix metalloproteinases prevents cardiac rupture but impairs therapeutic angiogenesis and causes cardiac failure. Nat Med 1999:5:1135-1142.

11. Wirtz $S$, Neufert $C$, Weigmann $B$, et al. Chemically induced mouse models of intestinal inflammation. Nat Protoc 2007;2:541-546.

12. Van der Sluis M, De Koning B, De Bruijn A, et al. Muc2-deficient mice spontaneously develop colitis, indicating that MUC2 is critical for colonic protection. Gastroenterology 2006;131:117-129.

13. Bradley PP, Priebat DA, Christensen RD, et al. Measurement of cutaneous inflammation: estimation of neutrophil content with an enzyme marker. J Invest Dermatol 1982;78:206-209.

14. Overbergh $L$, Giulietti $A$, Valckx D, et al. The use of real-time reverse transcriptase PCR for the quantification of cytokine gene expression. J Biomol Tech 2003;14:33-43.

15. Taylor CT, Colgan SP. Hypoxia and gastrointestinal disease. J Mol Med 2007;85:1295-1300.

16. Sakuraba $\mathrm{H}$, Ishiguro $\mathrm{Y}$, Yamagata $\mathrm{K}$, et al. Blockade of TGF- $\beta$ accelerates mucosal destruction through epithelial cell apoptosis. Biochem Biophys Res Commun 2007;359:406-412.

17. Spencer AU, Yang $\mathrm{H}$, Haxhija EQ, et al. Reduced severity of a mouse colitis model with angiotensin converting enzyme inhibition. Dig Dis Sci 2007;52:1060-1070.

18. Vetuschi A, Latella G, Sferra R, et al. Increased proliferation and apoptosis of colonic epithelial cells in dextran sulfate sodium-induced colitis in rats. Dig Dis Sci 2002;47:1447-1457.

19. Boismenu R, Chen Y, Chou K, et al. Orally administered RDP58 reduces the severity of dextran sodium sulphate induced colitis. Ann Rheum Dis 2002;61(Suppl 2):19-24.

20. Karhausen J, Furuta GT, Tomaszewski JE, et al. Epithelial hypoxiainducible factor- 1 is protective in murine experimental colitis. J Clin Invest 2004;114:1098-1106.

21. Ikeda $H$, Suzuki $Y$, Suzuki $M$, et al. Apoptosis is a major mode of cell death caused by ischaemia and ischaemia/reperfusion injury to the rat intestinal epithelium. Gut 1998;42:530-537.

22. Morote-Garcia JC, Rosenberger P, Nivillac NM, et al. Hypoxia-inducible factor-dependent repression of equilibrative nucleoside transporter 2 attenuates mucosal inflammation during intestinal hypoxia. Gastroenterology 2009;136:607-618. 
23. Fischer $C$, Mazzone $M$, Jonckx $B$, et al. FLT1 and its ligands VEGFB and PIGF: drug targets for anti-angiogenic therapy? Nat Rev Cancer 2008:8:942-956.

24. Kader HA, Tchernev VT, Satyaraj E, et al. Protein microarray analysis of disease activity in pediatric inflammatory bowel disease demonstrates elevated serum PLGF, IL-7, TGF-beta1, and IL-12p40 levels in Crohn's disease and ulcerative colitis patients in remission versus active disease. Am J Gastroenterol 2005;100:414-423.

25. Pousa ID, Maté J, Salcedo-Mora X, et al. Role of vascular endothelial growth factor and angiopoietin systems in serum of Crohn's disease patients. Inflamm Bowel Dis 2008;14:61-67.
26. Chidlow JH, Langston W, Greer JJM, et al. Differential angiogenic regulation of experimental colitis. Am J Pathol 2006;169:2014-2030.

27. Cooper H, Murthy SNS, Shah R, et al. Clinicopathologic study of dextran sulphate sodium experimental murine colitis. Laborat Invest 1993;69:238-249.

28. Asseman C, Mauze S, Leach MW, et al. An essential role for interleukin 10 in the function of regulatory $T$ cells that inhibit intestinal inflammation. J Exp Med 1999;190:995-1004.

29. Qualls JE, Kaplan AM, van Rooijen NJ, et al. Suppression of experimental colitis by intestinal mononuclear phagocytes. Leukoc Biol 2006;80:802-815 